\title{
Proton Magnetic Resonance Spectroscopy in 22q11 Deletion Syndrome
}

\author{
Fabiana da Silva Alves ${ }^{1 *}$, Erik Boot ${ }^{1,2}$, Nicole Schmitz ${ }^{1}$, Aart Nederveen ${ }^{3}$, Jacob Vorstman ${ }^{4}$, Christina \\ Lavini $^{3}$, Petra Pouwels ${ }^{5}$, Lieuwe de Haan ${ }^{1}$, Don Linszen ${ }^{1}$, Therese van Amelsvoort ${ }^{1,6}$
}

1 Department of Psychiatry, Academic Medical Centre Amsterdam, Amsterdam, The Netherlands, 2 Ipse de Bruggen, Centre for People with Intellectual Disability, Zwammerdam, The Netherlands, 3 Department of Radiology, Academic Medical Centre Amsterdam, Amsterdam, The Netherlands, 4 Department of Psychiatry, Rudolf Magnus Institute of Neuroscience, University Medical Centre Utrecht, Utrecht, The Netherlands, $\mathbf{5}$ Department of Physics and Medical Technology, VU University Medical Centre Amsterdam, Amsterdam, The Netherlands, 6 Arkin Mental Health Care, Amsterdam, The Netherlands

\begin{abstract}
Objective: People with velo-cardio-facial syndrome or 22q11 deletion syndrome (22q11DS) have behavioral, cognitive and psychiatric problems. Approximately $30 \%$ of affected individuals develop schizophrenia-like psychosis. Glutamate dysfunction is thought to play a crucial role in schizophrenia. However, it is unknown if and how the glutamate system is altered in 22q11DS. People with 22q11DS are vulnerable for haploinsufficiency of PRODH, a gene that codes for an enzyme converting proline into glutamate. Therefore, it can be hypothesized that glutamatergic abnormalities may be present in 22q11DS.

Method: We employed proton magnetic resonance spectroscopy ('H-MRS) to quantify glutamate and other neurometabolites in the dorsolateral prefrontal cortex (DLPFC) and hippocampus of 22 adults with 22q11DS (22q11DS SCZ+) and without (22q11DS SCZ-) schizophrenia and 23 age-matched healthy controls. Also, plasma proline levels were determined in the 22q11DS group.

Results: We found significantly increased concentrations of glutamate and myo-inositol in the hippocampal region of 22q11DS SCZ+ compared to 22q11DS SCZ-. There were no significant differences in levels of plasma proline between 22q11DS SCZ+ and 22q11DS SCZ-. There was no relationship between plasma proline and cerebral glutamate in 22q11DS.

Conclusion: This is the first in vivo ${ }^{1} \mathrm{H}-\mathrm{MRS}$ study in 22q11DS. Our results suggest vulnerability of the hippocampus in the psychopathology of 22q11DS SCZ+. Altered hippocampal glutamate and myo-inositol metabolism may partially explain the psychotic symptoms and cognitive impairments seen in this group of patients.
\end{abstract}

Citation: da Silva Alves F, Boot E, Schmitz N, Nederveen A, Vorstman J, et al. (2011) Proton Magnetic Resonance Spectroscopy in 22q11 Deletion Syndrome. PLoS ONE 6(6): e21685. doi:10.1371/journal.pone.0021685

Editor: Takeo Yoshikawa, Rikagaku Kenkyūsho Brain Science Institute, Japan

Received December 23, 2010; Accepted June 7, 2011; Published June 30, 2011

Copyright: (c) 2011 da Silva Alves et al. This is an open-access article distributed under the terms of the Creative Commons Attribution License, which permits unrestricted use, distribution, and reproduction in any medium, provided the original author and source are credited.

Funding: The study was funded by the Dutch Brain Foundation. The funders had no role in study design, data collection and analysis, decision to publish, or preparation of the manuscript.

Competing Interests: The authors have declared that no competing interests exist.

* E-mail: F.daSilvaAlves@amc.nl

\section{Introduction}

Velo-cadio-facial-syndrome or 22q11 deletion syndrome (22q11DS) is a genetic syndrome caused by a deletion on chromosome 22 which is accompanied by several somatic, behavioral, cognitive and psychiatric problems, and structural and functional brain abnormalities [1]. The estimated prevalence of 22q11DS in the general population is 1 in 5950 births [2]. Adults with 22q11DS face a 25 times higher risk of developing schizophrenia than the general population [3] and in people with schizophrenia an increased frequency of 22q11 deletions has been reported [4,5]. Hence, a 22q11 deletion is among the highest risk factors for the development of schizophrenia.

People with 22q11DS are vulnerable to haploinsufficiency of approximately 30 genes located on the deleted region of chromosome $22 q 11$, including the proline dehydrogenase gene (PRODH) [6]. This gene, which encodes for the PRODH enzyme also called proline oxidase (POX), is involved in converting proline to glutamate [7]. Dysfunction or genetic variations of the PRODH gene, and consequent hyperprolinemia, have been associated with susceptibility to schizophrenia and with learning disabilities [813]. In fact, proline has been shown to function as modulator of glutamate neurotransmission through NMDA receptors [14,15] and dysregulation of the glutamatergic system has been widely implicated in schizophrenia.

The involvement of glutamate in schizophrenia is particularly related to NMDA receptor hypofunction. Evidence for the role of NMDA receptor hypofunction in schizophrenia comes from pharmacological studies of phencyclidine (PCP) and ketamine. These NMDA receptor antagonists have shown to produce schizophrenia-like behaviors in rodents [16]; to induce positive and negative symptoms in healthy humans [17]; and to aggravate psychotic symptoms in patients with schizophrenia [18]. Glutamate also plays a role in synaptic plasticity via NMDA receptors mediating higher cognitive functions such as learning and memory. NMDA receptor dysfunction has also been implicated 
in the cognitive deficits of schizophrenia [19]. In these people, agents that enhance NMDA receptor activity have shown to improve negative symptoms and to facilitate memory consolidation [20].

The brain areas associated with NMDA receptor hypofunction in schizophrenia include the prefrontal cortex and hippocampus [21-24]. The relationship between NMDA receptor hypofunction and glutamate release is not fully understood. NMDA hypofunction in schizophrenia could be related to insufficient or excessive glutamate release which may also differ between brain regions [25]. Increased glutamate exposure and its duration could explain the psychotoxic effects in schizophrenia.

Proton Magnetic Resonance Spectroscopy $\left({ }^{1} \mathrm{H}-\mathrm{MRS}\right)$ is a feasible method for in vivo quantification of glutamate concentration and other brain metabolites that, if altered, may reflect abnormal neuro-developmental features [26]. In schizophrenia an increasing number of ${ }^{1} \mathrm{H}-\mathrm{MRS}$ studies have been conducted. Although inconclusive, ${ }^{1} \mathrm{H}-\mathrm{MRS}$ findings also suggest abnormal glutamatergic neurotransmission [27-29].

To date, the glutamatergic system in 22q11DS has not been investigated. People with 22q11DS have an increased prevalence of schizophrenia and similar neuroanatomical abnormalities. Hence, in this study we employed ${ }^{1} \mathrm{H}-\mathrm{MRS}$ to measure glutamate in the dorsolateral prefrontal cortex and hippocampus in 22q11DS patients with (22q11DS SCZ+) and without schizophrenia (22q11DS SCZ-). We hypothesized altered glutamate concentrations in individuals with 22q11DS SCZ+ compared to healthy individuals and, in 22q11DS SCZ+ compared to 22q11DS SCZ-. Besides glutamate, we also analyzed other neurometabolites from ${ }^{1} \mathrm{H}-\mathrm{MRS}$ spectra including $\mathcal{N}$-acetylaspartate, choline, myoinositol and creatine which reflect the status of neuronal functioning and glial cells, possibly disturbed in 22q11DS.

Furthermore, we assessed levels of plasma proline and plasma glutamine in the $22 q 11 \mathrm{DS}$ group. Increased proline has been reported in 22q11DS patients [30]. In children with 22q11DS there was a relationship between increased plasma proline and decreased brain function [31]. High levels of proline in 22q11DS, consequence of POX deficiency, may be related to glutamate dysfunction particularly in 22q11DS SCZ+. Hence, we expected that plasma proline will be increased in 22q11DS SCZ+ and that it will correlate with glutamate concentrations in the brain.

\section{Materials and Methods}

\section{Subjects}

We included 22 adults with 22q11DS (mean \pm SD) (22q11DS SCZ $+n=12$, age $29.25 \pm 8.24$; 22q11DS SCZ $-n=10$, age $28.50 \pm 8.47$ ) and 23 healthy controls (HC, age 31.22 \pm 9.58$)$.

Individuals with 22q11DS were recruited through the Dutch 22q11DS family association and through the departments of three Dutch Clinical Genetics centers. Healthy volunteers were recruited by local advertisement. The study was conducted at the Department of Psychiatry, Academic Medical Centre Amsterdam (AMC), The Netherlands and was approved by the Medical Ethics Testing Committee/AMC. All participants were capable of giving written informed consent and did so, after receiving full information on the study.

All individuals with 22q11DS were assessed by an experienced psychiatrist and a physician for people with an intellectual disabilities using available information from medical records and a semi-structured psychiatric interview. All diagnoses reported are DSM-IV diagnoses (American Psychiatric Association, 1994). The 22q11DS group was subdivided into 2 groups: those who were fulfilling DSM-IV criteria for schizophrenia (22q11DS SCZ+) all taking antipsychotic medication and having duration of illness $>1$ year (dose ranges and haloperidol equivalents [32] are displayed in Table 1) and those who did not have a past or current psychiatric history and had never taken antipsychotic or stimulant medication (22q11DS SCZ-).

In addition, the Positive and Negative Symptom Scale (PANSS) [33] was used to assess positive, negative and general psychopathology in the 22q11DS SCZ+ group. The PANSS includes 30 items, subdivided in three categories: positive symptoms, negative symptoms and general psychopathology. A patient who rates "absent" (or 1) on all items would receive a total score of 30 and a subject who rates "extreme" (or 7) on all 30 items would receive a total score of 210. All patients underwent a formalized clinical interview of 35-40 minutes and the questions were in regard to the last two weeks.

For assessment of intelligence quotient (IQ) we used the shortened Dutch version of the Wechsler Adult Intelligence Scale (WAIS-III-NL) consisting of 5 subtests: vocabulary, comprehension, similarities (verbal IQ), block design, and object assembly (performance IQ $[34,35]$.

All healthy volunteers were seen by a physician. They were included in the study after screening for psychiatric disorders and medical conditions affecting the brain. None of the participants had a history of substance or alcohol abuse. Urine drug screening (cocaine, tetrahydrocannabinol, opiates, amphetamines, benzodiazepines) was performed at study day and was negative in all subjects. Healthy participants were not using any medication at the time of testing.

\section{H-MR spectroscopy acquisition}

${ }^{1}$ H-MRS data acquisition took place at the Department of Radiology (Academic Medical Centre Amsterdam, The Netherlands) using a 3 Tesla Intera MRI system (Philips, Best, The Netherlands) equipped with a 6 channel sense head coil. For estimation of metabolite concentrations, two single $8 \mathrm{ml}$ voxels of interest positioned in the left dorsolateral prefrontal cortex (DLPFG) $(2 \times 2 \times 2 \mathrm{~cm})$ and left hippocampus $(2 \times 2 \times 2 \mathrm{~cm})$ were obtained for each subject (Figure 1). More specifically, the hippocampal voxel included areas of the hippocampus, parahippocampal gyrus, fusiform gyrus and collateral sulcus. Iterative first order shimming was performed and water suppressed spectra was acquired using a point-resolved spatially localized spectroscopy sequence (PRESS, TE 36 ms, TR 2000 ms, 128 averages).

Table 1. Medication and dosage taken by 22q11DS patients with schizophrenia.

\begin{tabular}{|c|c|c|c|}
\hline Drugs & Dosis (mg/d) & $\begin{array}{l}\text { Haloperidol } \\
\text { equivalent }(\mathrm{mg} / \mathrm{d})^{\mathrm{a}}\end{array}$ & $\mathbf{N}$ \\
\hline Aripiprazole & $5-15$ & $1-7.5$ & 3 \\
\hline Atomoxetine $^{b}$ & 80 & & 1 \\
\hline Clozapine & $200-300$ & $4-6$ & 2 \\
\hline Methylphenidate $^{c}$ & 36 & & 1 \\
\hline Olanzapine & 5 & 2.5 & 1 \\
\hline Quetiapine & 50 & 0.5 & 2 \\
\hline Risperidone & $3-4$ & $5-6.7$ & 2 \\
\hline Zuclopentixol & 6 & 1.2 & 1 \\
\hline
\end{tabular}

${ }^{\mathrm{a}}$ Haloperidol equivalents derived from kane et al (2003).

${ }^{b}$ One patient took an antipsychotic and a selective norepinephrine inhibitor.

'One patient took an antipsychotic and a psychostimulant drug.

doi:10.1371/journal.pone.0021685.t001 


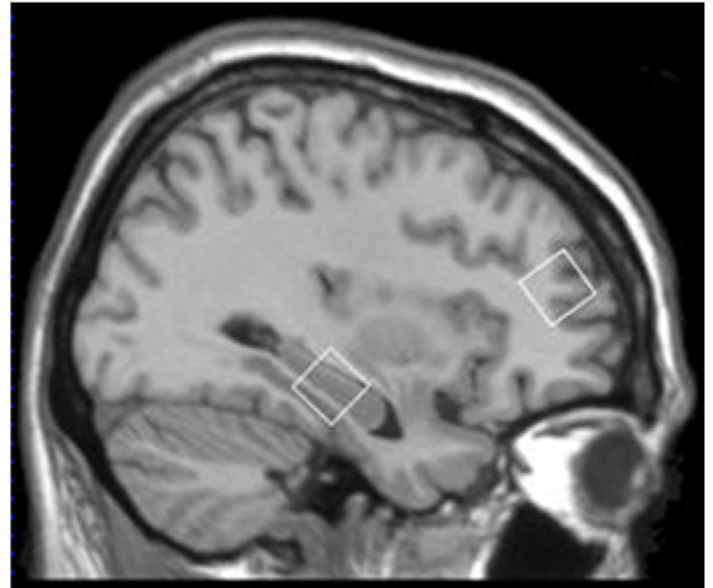

Figure 1. Sargittal T1-weighted magnetic resonance image of the brain showing voxel $(2 \times 2 \times 2 \mathrm{~cm})$ placement for proton magnetic resonance spectroscopy (1H-MRS) in the left dorsolateral prefrontal cortex and left hippocampus.

doi:10.1371/journal.pone.0021685.g001

For anatomical localization transversal high-resolution structural T1-weighted volumetric images, with full head coverage, using 130 contiguous slices $(1.2 \mathrm{~mm}$ thick, with $0.89 \times 0.89 \mathrm{~mm}$ in-plane resolution) and a TR/TE of 9.8/4.5 milliseconds (flip angle 8", FOV $224 \mathrm{~cm}$ ) were obtained.

${ }^{1}$ H-MRS spectra were analyzed using the Linear Combination of Model spectra (LCModel) commercial spectral-fitting package [36]. LCModel used a library of reference spectra in a basis set recorded specifically for the scanner and calibrated using the tissue water signal as an internal standard. The spectra were analyzed with a range of $3.8 \mathrm{ppm}$ to $0.2 \mathrm{ppm}$ (Figure 2). From the metabolites included in the LCModel basis set, we analyzed absolute levels of creatine plus phosphocreatine (Cr), glycerophosphocholine plus phosphocholine (choline), myo-inositol, $\mathcal{N}$-acetylaspartate (NAA), NAA plus $\mathcal{N}$-acetylaspartylglutamate (NAAG), glutamine and glutamate.

In addition, we analyzed the combination of glutamate plus glutamine (Glx). Glutamate and glutamine are closely related amino acids involved in intermediary metabolism, protein synthesis and neurotransmission. Metabolite concentrations are expressed in millimoles per liter.

Data were excluded from analysis if the voxel coordinates were not or incorrectly recorded. Spectral width (full width at half maximum, FWHM) was always lower than 0.1 p.p.m. and signal to noise ratio (SNR) greater than 11 as estimated by LCModel. Cramer-Rao minimum variance bounds (SD) was lower than $50 \%$ for glutamine and lower than $15 \%$ for the other metabolites.

\section{Plasma amino-acid analyses}

Plasma proline and plasma glutamine concentrations of the 22q11DS group were assessed by automated ion exchange chromatography with post-column ninhydrin derivatization. Plasma amino-acid analyses were performed on a JEOL AminoTac (JEOL AminoTac JLC-500/V, Tokyo, Japan) following a morning blood draw.

\section{Statistical analyses}

We used non-parametric Kruskal-Wallis $\mathrm{H}$ test to compare metabolite concentrations, age and IQ between the 3 groups $(\mathrm{HC}$, 22q11DS SCZ+ and 22q11DS SCZ-) because the assumption of normal distribution was not met. Following, Post Hoc analyses were conducted with Mann-Whitney U tests. Correlation analyses were conducted with Spearman's rho test. Results are reported as significant when $P \leq 0.05$ (2-tailed). Statistical analyses were performed with SPSS, release 16.0.2 for Windows (SPSS Inc., Chicago, IL, USA. 2008).

\section{Results}

\section{Demographics}

Patients and healthy controls did not differ with regard to sex (HC 12m/11f, 22ql1DS SCZ+ 8m/4f, 22q11DS SCZ- 4m/6f $P=0.45)$ and age (HC 31.22 $\pm 9.51,22 q 11 \mathrm{DS}$ SCZ+ age 29.25 $\pm 8.24,22 q 11 \mathrm{DS}$ SCZ $-28.50 \pm 8.47 ; P=0.89$ ).

Patients had a lower total IQ than healthy controls (HC $111.88 \pm 14.82,22 q 11 \mathrm{DS} \mathrm{SCZ}+69.67 \pm 13.82,22 \mathrm{q} 11 \mathrm{DS} \mathrm{SCZ}-$

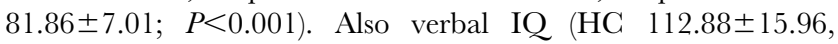

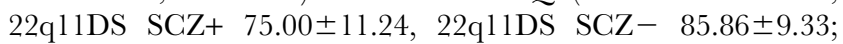
$P=0.001)$ and performance IQ (HC 109.38 $\pm 19.91,22 q 11 \mathrm{DS}$ $\mathrm{SCZ}+67.89 \pm 16.60,22 \mathrm{q11DS}$ SCZ $-79.43 \pm 10.53 ; P=0.002)$ were significantly different between the groups. Post hoc analysis showed that HC compared to 22q11DS SCZ+ differed significantly for total IQ $P=0.001$, verbal IQ $P=0.001$ and performance IQ $P=0.001$. HC compared to 22q11DS SCZ - differed significantly for total IQ $P=0.004$, verbal $\mathrm{IQ}=P=0.005$ and performance IQ $P=0.01$. 22q11DS SCZ+ compared to 22q11DS SCZ - differed significantly for total IQ $P=0.02$ and verbal IQ $P=0.02$ but not performance IQ $P=0.17$.

For the 22q11DS SCZ+ group, the mean score on the general psychopathology PANSS subscale was $30.69 \pm 11.94$, the negative subscale was $17.55 \pm 8.21$ and the positive subscale was $10.69 \pm 3.81$. The mean of total PANSS score was 58.95 \pm 21.85 .

\section{Metabolites}

Metabolite concentrations for the DLPFC and hippocampal region are displayed in Table 2. Kruskal-Wallis $\mathrm{H}$ test showed no significant group differences in any of the metabolite concentrations in the DLPFC. In the hippocampal region, significant group differences were found in concentrations of glutamate $(P=0.03)$ Glx $(P=0.03)$ and myo-inositol $(P=0.03)$. Post Hoc analysis indicated that these metabolite concentrations were significantly higher in 22q11DS SCZ+ compared to 22q11DS SCZ- patients (glutamate $P=0.02$; Glx $P=0.03$ and myo-inositol $P=0.01$ ). Hippocampal Glx was higher in 22q1 1DS SCZ+ compared to HC $(P=0.02)$. In the DLPFC there was a significant positive correlation between glutamine concentration and antipsychotic dosage $(n=10 \rho=0.64 P=0.05)$ and a trend towards a positive correlation between Glx and antipsychotic dosage $(\mathrm{n}=10 \rho=0.59$ $P=0.07)$. There were no significant correlations between hippocampal metabolites and antipsychotic dosage.

\section{Plasma Proline and Plasma Glutamine}

For the whole 22q11DS group, the mean \pm SD for plasma proline was $\mathrm{n}=13,354 \pm 128.88 \mu \mathrm{mol} / \mathrm{l}$ and for plasma glutamine $\mathrm{n}=8,540.62 \pm 68.14 \mu \mathrm{mol} / \mathrm{l}$. The correlation between these variables was not significant $(n=8 \rho=0.26 P=0.53)$. The normal laboratory range for plasma proline was $77-343 \mu \mathrm{mol} / \mathrm{l}$ and for plasma glutamine $344-743 \mu \mathrm{mol} / \mathrm{l}$.

There were no significant differences between 22q11DS SCZand 22q11DS SCZ+ for plasma proline (22q11DS SCZ- $\mathrm{n}=8$, $376.37 \pm 145.64 \mu \mathrm{mol} / \mathrm{l}, 22 q 1 \mathrm{DS}$ SCZ+ $\mathrm{n}=5, \quad 318.20 \pm 100.56$ $\mu \mathrm{mol} / \mathrm{l} ; \quad P=0.56)$ or plasma glutamine (22q11DS SCZ $-\mathrm{n}=4$, $555.25 \pm 79.47 \mu \mathrm{mol} / \mathrm{l}, 22 q 11 \mathrm{DS} \mathrm{SCZ}+\mathrm{n}=5,540.80 \pm 63.70 \mu \mathrm{mol} /$ 


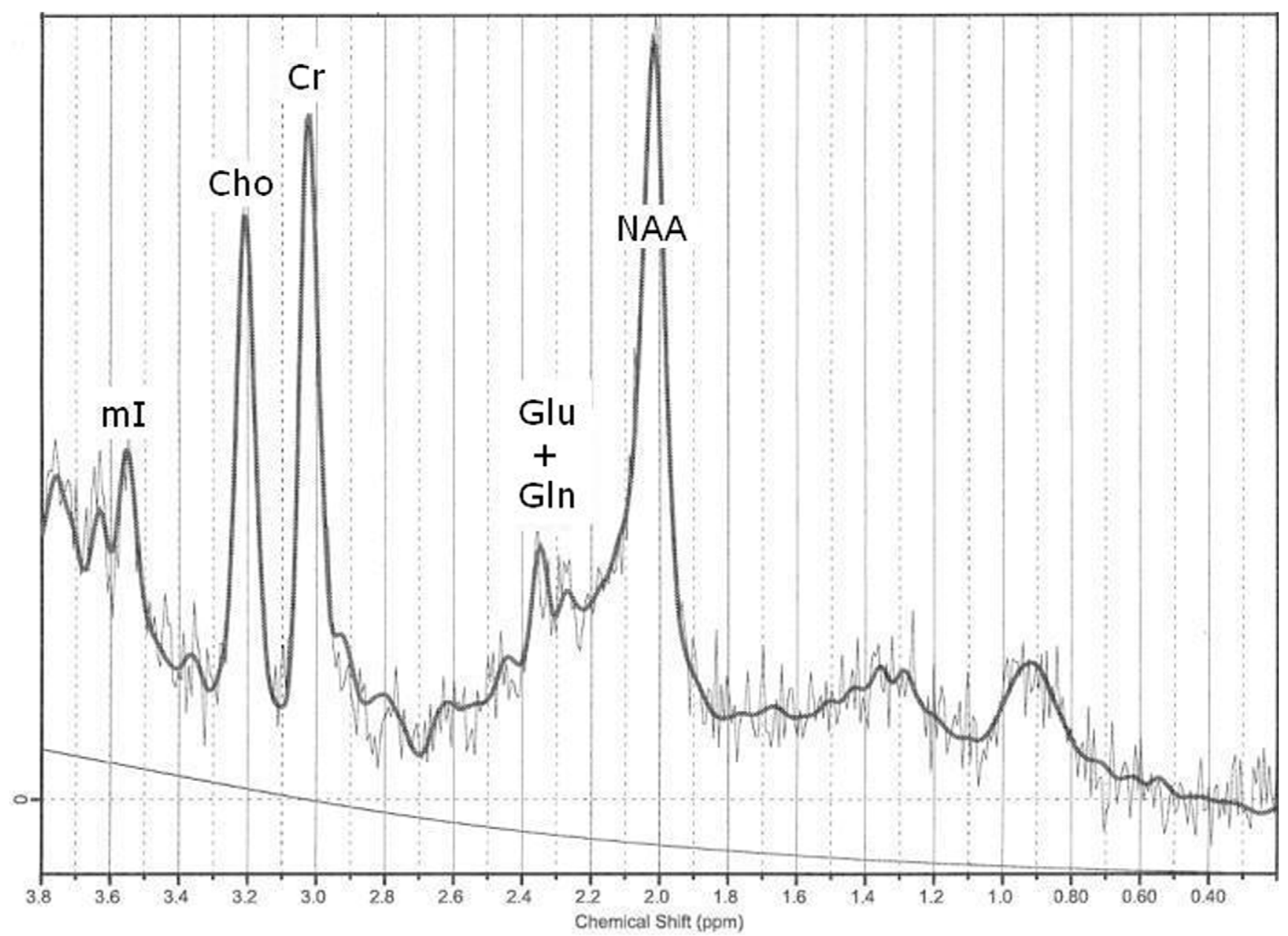

Figure 2. Sample of a 1H-MRS spectrum from hippocampus of a patient with 22q11DS as fit by LCModel. doi:10.1371/journal.pone.0021685.g002

Table 2. Metabolites concentrations (mean/SD) in the DLPFC and hippocampal region in healthy controls and 22q11DS with and without psychosis.

\begin{tabular}{|c|c|c|c|c|c|c|c|}
\hline DLPFC & $H C$ & $S C Z-$ & $S C Z+$ & HIP & $H C$ & $S C Z-$ & $S C Z+$ \\
\hline$n=$ & 23 & 7 & 11 & $n=$ & 16 & 7 & 9 \\
\hline Glu & $6.44 / 1.35$ & $6.35 / 1.02$ & $6.39 / 1.32$ & $\mathrm{Glu}^{\mathrm{a}}$ & $6.26 / 0.65$ & $5.71 / 0.94$ & $6.99 / 1.04$ \\
\hline Gln & $2.86 / 0.94$ & $2.66 / 0.83$ & $3.25 / 1.37$ & Gln & $3.03 / 0.83$ & $3.12 / 0.58$ & $3.88 / 1.67$ \\
\hline Glx & $9.17 / 2.06$ & $8.64 / 1.29$ & $9.65 / 2.28$ & $\mathrm{Gl}^{\mathrm{ab}}$ & $9.29 / 0.94$ & $8.83 / 1.11$ & $10.87 / 1.66$ \\
\hline $\mathrm{ml}$ & $3.51 / 0.54$ & $3.35 / 0.50$ & $3.46 / 0.83$ & $\mathrm{ml}^{\mathrm{a}}$ & $3.87 / 0.63$ & $3.47 / 0.40$ & $4.43 / 0.76$ \\
\hline NAA & $6.07 / 0.79$ & $5.38 / 0.63$ & $5.89 / 0.82$ & NAA & $5.03 / 0.57$ & $4.63 / 0.85$ & $5.25 / 1.18$ \\
\hline NAA+NAAG & $6.68 / 0.82$ & $5.96 / 0.92$ & $6.41 / 1.11$ & $\mathrm{NAA+NAAG}$ & $5.64 / 0.75$ & $5.44 / 0.72$ & $6.06 / 1.09$ \\
\hline Cho & $1.38 / 0.16$ & $1.34 / 0.22$ & $1.43 / 0.20$ & Cho & $1.58 / 0.18$ & $1.54 / 0.17$ & $1.71 / 0.25$ \\
\hline $\mathrm{Cr}$ & $5.06 / 0.60$ & $4.80 / 0.38$ & $5.06 / 0.60$ & $\mathrm{Cr}$ & $4.96 / 0.54$ & $4.70 / 0.64$ & $5.25 / 0.86$ \\
\hline
\end{tabular}

HC: Healthy controls SCZ-: 22q11DS without psychosis SCZ+: 22q11DS with psychosis.

Glu: glutamate Gln: glutamine Glx: Glu+Gln NAA: $N$-acetylaspartate.

NAA+NAAG: NAA+N-acetylaspartylglutamate ml: myo-inositol Cr: creatine Cho: choline.

Metabolite concentrations are expressed in millimoles per liter.

${ }^{\mathrm{a}} P<0.05$ for SCZ - vs. SCZ+.

${ }^{\mathrm{b}} P=0.05$ for $\mathrm{HC}$ vs. SCZ+.

doi:10.1371/journal.pone.0021685.t002 
1; $P=0.78$ ). There was no significant correlation between plasma proline and plasma glutamine in any of the two 22q11DS groups.

The correlation between DLPFC glutamate and plasma proline for the whole 22q11DS group was not significant $(\mathrm{n}=11 \rho=0.26$ $P=0.43)$. Also, there was no significant correlation between proline and DLPFC glutamate for the 22q11DS SCZ- $(n=5$ $\rho=0.30 P=0.62)$ and 22q11DS SCZ+ group $(\mathrm{n}=6 \rho=0.37$ $P=0.47)$.

The correlation between hippocampal glutamate and plasma proline for the whole 22q11DS group was not significant $(\mathrm{n}=10$ $\rho=0.21 P=0.56$ ). There was no significant correlation between proline and hippocampal glutamate for the 22q11DS SCZ$(\mathrm{n}=6 \quad \rho=0.03 \quad P=0.96)$ and $22 q 11 \mathrm{DS}$ SCZ+ group $(\mathrm{n}=4$ $\rho=0.40 P=0.80)$.

\section{Discussion}

In this first in vivo ${ }^{1} \mathrm{H}-\mathrm{MRS}$ study in 22q11DS we measured metabolite concentrations of the DLPFC and hippocampal region in adults with and without schizophrenia and in healthy controls. Our main findings are increased hippocampal glutamate and myoinositol concentrations in 22q11DS SCZ+ compared to 22q11DS SCZ - . Metabolites of the DLPFC did not differ significantly across the groups.

${ }^{1} \mathrm{H}-\mathrm{MRS}$ studies of the hippocampus in schizophrenia have shown ambivalent results concerning glutamate; some studies reported no alterations of glutamate concentrations in subjects experiencing prodromal symptoms of schizophrenia [37] or in chronic schizophrenia $[38,39]$. Other studies reported increased hippocampal glutamate in patients with schizophrenia [40] or a tendency towards increased glutamate in a group of medicated first episode patients [41].

In the present ${ }^{1} \mathrm{H}-\mathrm{MRS}$ study we found increased concentration of glutamate and Glx in the hippocampal region of 22q11DS SCZ+ compared to 22q11DS SCZ-. Also, hippocampal Glx was increased in 22ql1DS SCZ+ compared to healthy controls. Excessive release of glutamate and consequent overstimulation of postsynaptic receptors might have an influence on the cognitive and psychotic symptoms associated with the NMDA hypofunction in schizophrenia [25]. In line with this observation and in agreement with previous research in schizophrenia, our finding of increased hippocampal glutamate in 22q11DS SCZ+ suggests that glutamate disturbance may be underlying psychotic symptoms in 22q11DS SCZ+. Moreover, the 22q11DS SCZ+ had overall lower IQ than 22q11DS SCZ-. Increased hippocampal glutamate could also explain the cognitive impairment in 22q11DS SCZ+ since this brain area is involved in learning and memory functions. Although speculative, increased hippocampal glutamate in 22q11DS SCZ+ might also indicate NMDA receptor hypofunction in this group.

Glutamate neurotransmission may in part be influenced by proline. Increased concentrations of proline associated with hyperprolinemia type II (proline levels 10-15 fold above normal) have been shown to potentiate glutamate transmission in hippocampus and cerebral cortex [15,42]. Hyperprolinemia of the type I has been observed in patients with 22q11DS (plasma proline levels with a range of 3-10 fold above normal) which results from inherited deficiency of POX enzyme [12,30]. In the present study half of the 22q11DS patients had elevated proline levels. Contrary to our expectation, we found similar proline levels in 22q11DS SCZ+ and 22q11DS SCZ-. Increased proline levels may depend on genetic variation of the PRODH allele [8] or on interaction with other genes. For instance, a study of hyperprolinemia in 22q11DS showed an association between hyperprolin- emia and psychosis in 22q11DS patients only when Met, the low activity allele of the COMT gene, was taken into account [12]. We found no correlation between plasma proline, plasma glutamine and cerebral glutamate concentrations in the whole 22q11DS group or in 22q11DS SCZ - vs. 22q11DS SCZ+. Thus, although we found increased hippocampal glutamate concentrations in 22q11DS SCZ+, its underlying mechanisms remain unclear.

In addition to increased hippocampal glutamate, we found higher concentrations of myo-inositol in 22q11DS SCZ+ compared to 22q11DS SCZ-. Increased concentrations of myo-inositol have previously been reported in mild cognitive impairment and Alzheimer disease [43,44]. Also in Down syndrome increased hippocampal myo-inositol has been associated with reduced cognitive ability [45]. Changes in myo-inositol levels may reflect abnormalities in membrane metabolism, in intracellular signaling mechanisms, neuronal development and survival [46]. Hence, increased myo-inositol may explain part of the hippocampal brain abnormalities and learning disabilities seen in 22q11DS SCZ+.

The finding of increased glutamate and myo-inositol may be tightly related to each other in the psychopathology in 22q11DS $\mathrm{SCZ}+$. Myo-inositol is primarily found in astrocytes [47] which interact with neurons and play a critical role in the synthesis of glutamate $[48,49]$. Elevated concentration of myo-inositol may indicate increased number or increased metabolic activity of astrocytes. Astrocyte dsysregulation in turn may trigger increased glutamate uptake and glutamate-glutamine cycling conversion. This could reflect altered glutamatergic neurotransmission in this genetic predisposed group, which combined with environmental interaction may increase the vulnerability for development of schizophrenia.

We found no significant variation in neurometabolites concentration between the whole 22q11DS patient group and the healthy control group. This might be explained by group differences in the proportion of gray matter/white matter within the DLPFC and hippocampal voxels. Also, we found no evidence for altered glutamate in the DLPFC of 22q11DS patients (22q11DS SCZ+ vs. 22q11DS SCZ-) vs. healthy controls. In patients with chronic schizophrenia, ${ }^{1} \mathrm{H}-\mathrm{MRS}$ studies of the frontal cortex have shown increased [40,50,51] and reduced glutamate concentrations $[29,39,52,53]$. Perhaps, brain dysfunction associated with psychosis in 22q11DS involves specific regions of the temporal lobe $[54,55]$. Furthermore, it is also possible that abnormalities in glutamatergic function in this brain region may exist at the level of NMDA receptor or in second messenger signaling without alterations in glutamate concentration.

An interesting observation is that most of the metabolite contents are in the order of 22q11DS SCZ $-<\mathrm{HC}<22 q 11 \mathrm{DS}$ SCZ+. We are not aware of an existing explanation for this relation in the literature. However, we hypothesize that prior to the development of schizophrenia patients with 22q11DS in general may have decreased neuronal metabolism as has been observed for glutamate in individuals with increased vulnerability to schizophrenia (at risk mental state - ARMS) [56,57]. On the other hand, an instable neuronal metabolism may predispose a subgroup of 22q11DS patients to psychotic decompensation. Another possibility is that higher metabolites in the 22q11DS patients are the result of the transition to psychosis instead of the cause. This would mean that high metabolic rates in 22q11DS are state- instead of trait-related. Due to the cross-sectional design of our study we are unable to confirm this hypothesis. Longitudinal research in 22q11DS patients before and after transition to psychosis is therefore warranted.

The strengths of this study include the evaluation of neuronal integrity in 22q11DS according to psychiatric status of 22q11DS 
SCZ - and 22q11DS SCZ+ and in comparison to age matched healthy controls. Also, all MRS spectra were carefully inspected and were included only if fulfilling the quality criteria of LCmodel.

We have to acknowledge some limitations of the study; unfortunately at the time of the study we were not able to analyze plasma samples of proline and glutamine of healthy controls. Future studies with large sample sizes including healthy volunteers, should elucidate the relationship between plasma levels (of proline, glutamate, glutamine), cerebral metabolites and the vulnerability to schizophrenia. We did not determine the size of deleted region in each 22q11DS patient although the majority of patients have a typically deleted $3 \mathrm{Mb}$ region. We did not to apply a correction for multiple comparisons because the possibility of inflating type II error [58]. Since increased hippocampal glutamate possibly corroborates the involvement of glutamate in psychosis [11,41,59] and converging evidence from animal and human studies propose the hippocampus as crucial brain area involved in the vulnerability to schizophrenia $[60,61]$ we chose to avoid a too stringent evaluation. We were not able to determine tissue contributions to measured metabolites; the use of unsegmented voxels (i.e., assessment of metabolite concentrations without addressing the impact of different tissue included in the voxel of interest) may increase the standard error of measurement and diminish the power to detect significant differences. The cubic shape of hippocampal voxel may have allowed for contamination signals from adjacent regions of the hippocampus. Moreover, the effect of medication can be a potentially confounding factor in ${ }^{1} \mathrm{H}-\mathrm{MRS}$ studies [62]. In our study, antipsychotic drugs may have affected metabolites concentrations of frontal lobe in 22q11DS SCZ+. In fact, in the DLPFC, unlike in the hippocampus, we found a significant positive

\section{References}

1. Gothelf D, Schaer M, Eliez S (2008) Genes, brain development and psychiatric phenotypes in velo-cardio-facial syndrome. Dev Disabil Res Rev 14: 59-68.

2. Botto LD, May K, Fernhoff PM, Correa A, Coleman K, et al. (2003) A population-based study of the 22q11.2 deletion: phenotype, incidence, and contribution to major birth defects in the population. Pediatrics 112: 101-107.

3. Murphy KC, Jones LA, Owen MJ (1999) High rates of schizophrenia in adults with velo-cardio-facial syndrome. Arch Gen Psychiatry 56: 940-945.

4. Hoogendoorn ML, Vorstman JA, Jalali GR, Selten JP, Sinke RJ, et al. (2008) Prevalence of 22q11.2 deletions in 311 Dutch patients with schizophrenia. Schizophr Res 98: 84-88.

5. Stone LJ, O'Donovan MC, Gurling H, Kirov KG, Blackwood DHR, et al. (2008) Rare chromosomal deletions and duplications increase risk of schizophrenia. Nature 455: 237-241.

6. Lindsay EA (2001) Chromosomal microdeletions: dissecting del22q11 syndrome. Nat Rev Genet 2: 858-868.

7. Phang JM, Hu C-A, Valle D (2001) Disorders of proline and hydroxyproline metabolism. pp 1821-1838.

8. Bender HU, Almashanu S, Steel G, Hu CA, Lin WW, et al. (2005) Functional consequences of PRODH missense mutations. Am J Hum Genet 76: 409-420.

9. Jacquet H, Raux G, Thibaut F, Hecketsweiler B, Houy E, et al. (2002) PRODH mutations and hyperprolinemia in a subset of schizophrenic patients. Hum Mol Genet 11: 2243-2249.

10. Lui H, Heath SC, Sobin C, Roos JL, Galke BL, et al. (2002) Genetic variation at the 22q11 PRODH2/DGCR6 locus presents an unusual pattern and increases susceptibility to schizophrenia. Proc Natl Acad Sci USA 99: 3717-3722.

11. Paterlini M, Zakharenko SS, Lai WS, Qin J, Zhang H, et al. (2005) Transcriptional and behavioral interaction between 22q11.2 orthologs modulates schizophrenia-related phenotypes in mice. Nat Neurosci 8: 1586-1594.

12. Raux G, Bumsel E, Hecketsweiler B, van Amelsvoort T, Zinkstok J, et al. (2007) Involvement of hyperprolinemia in cognitive and psychiatric features of the 22q11 deletion syndrome. Hum Mol Genet 16: 83-91.

13. Willis A, Bender HU, Steel G, Valle D (2008) PRODH variants and risk for schizophrenia. Amino Acids 35: 673-679.

14. Cohen SM, Nadler JV (1997) Proline-induced inhibition of glutamate release in hippocampal area CA1. Brain Res 769: 333-339.

15. Cohen SM, Nadler JV (1997) Proline-induced potentiation of glutamate transmission. Brain Res 761: 271-282.

16. Arguello PA, Gogos JA (2006) Modeling madness in mice: one piece at a time. Neuron 52: 179-196.

17. Krystal JH, Karper LP, Seibyl JP, Freeman GK, Delaney R, et al. (1994) Subanesthetic effects of the noncompetitive NMDA antagonist, ketamine, in correlation between dosage of medication and glutamine concentration and a trend towards positive correlation between dosage of medication and Glx concentration in 22q11DS SCZ+ patients. This may also indicate that antipsychotics modulates neuronal metabolism in a regionally specific fashion.

Due to similar chemical components glutamate and glutamine overlap significantly in the ${ }^{1} \mathrm{H}$ resonance spectrum. The use of higher field strengths and implemented spectroscopy analysis technique makes it possible to improve glutamate quantification. Discrepancies across earlier ${ }^{1} \mathrm{H}-\mathrm{MRS}$ studies that proposed to investigate glutamate in psychosis could have resulted from differences in brain regions of interest, patient population and stage of disease or issues of spectroscopy measurements.

In conclusion, our findings suggest vulnerability of the hippocampus in the psychopathology of 22q11DS SCZ+. Although the generalizability of the results is restricted by the relatively small sample size, altered glutamate and myo-inositol metabolism may partially explain the psychotic symptoms and cognitive impairments seen in this group of patients. Future ${ }^{1} \mathrm{H}$ MRS studies with larger sample sizes including other prefrontal and temporal brain regions will help to clarify brain metabolism and integrity in $22 \mathrm{q} 11 \mathrm{DS}$.

\section{Author Contributions}

Conceived and designed the experiments: TvA NS FdSA. Performed the experiments: FdSA EB. Analyzed the data: FdSA CL PP AN EB. Contributed reagents/materials/analysis tools: JV AN CL. Wrote the paper: FdSA EB TvA NS LdH DL. humans. Psychotomimetic, perceptual, cognitive, and neuroendocrine responses. Arch Gen Psychiatry 51: 199-214.

18. Lahti AC, Koffel B, LaPorte D, Tamminga CA (1995) Subanesthetic doses of ketamine stimulate psychosis in schizophrenia. Neuropsychopharmacology 13: 9-19.

19. Moghaddam B (2004) Targeting metabotropic glutamate receptors for treatment of the cognitive symptoms of schizophrenia. Psychopharmacology (Berl) 174: 39-44.

20. Goff DC, Tsai G, Levitt J, Amico E, Manoach D, et al. (1999) A placebocontrolled trial of $\mathrm{D}$-cycloserine added to conventional neuroleptics in patients with schizophrenia. Arch Gen Psychiatry 56: 21-27.

21. Beneyto M, Kristiansen LV, Oni-Orisan A, McCullumsmith RE, MeadorWoodruff JH (2007) Abnormal glutamate receptor expression in the medial temporal lobe in schizophrenia and mood disorders. Neuropsychopharmacology 32: $1888-1902$

22. Burbaeva GS, Boksha IS, Turishcheva MS, Vorobyeva EA, Savushkina OK, et al. (2003) Glutamine synthetase and glutamate dehydrogenase in the prefrontal cortex of patients with schizophrenia. Prog Neuropsychopharmacol Biol Psychiatry 27: 675-680.

23. Harrison PJ, Weinberger DR (2005) Schizophrenia genes, gene expression, and neuropathology: on the matter of their convergence. Mol Psychiatry 10: 40-68.

24. Pilowsky LS, Bressan RA, Stone JM, Erlandsson K, Mulligan RS, et al. (2006) First in vivo evidence of an NMDA receptor deficit in medication-free schizophrenic patients. Mol Psychiatry 11: 118-119.

25. Olney JW, Newcomer JW, Farber NB (1999) NMDA receptor hypofunction model of schizophrenia. J Psychiatr Res 33: 523-533.

26. Soares DP, Law M (2009) Magnetic resonance spectroscopy of the brain: review of metabolites and clinical applications. Clin Radiol 64: 12-21.

27. Bartha R, Williamson PC, Drost DJ, Malla A, Carr TJ, et al. (1997) Measurement of glutamate and glutamine in the medial prefrontal cortex of never-treated schizophrenic patients and healthy controls by proton magnetic resonance spectroscopy. Arch Gen Psychiatry 54: 959-965.

28. Theberge J, Bartha R, Drost DJ, Menon RS, Malla A, et al. (2002) Glutamate and glutamine measured with $4.0 \mathrm{~T}$ proton MRS in never-treated patients with schizophrenia and healthy volunteers. Am J Psychiatry 159: 1944-1946.

29. Theberge J, Al-Semaan Y, Williamson PC, Menon RS, Neufeld RW, et al. (2003) Glutamate and glutamine in the anterior cingulate and thalamus of medicated patients with chronic schizophrenia and healthy comparison subjects measured with 4.0-T proton MRS. Am J Psychiatry 160: 2231-2233.

30. Goodman BK, Rutberg J, Lin WW, Pulver AE, Thomas GH (2000) Hyperprolinaemia in patients with deletion (22)(q11.2) syndrome. J Inherit Metab Dis 23: 847-848. 
31. Vorstman JA, Turetsky BI, Sijmens-Morcus ME, de Sain MG, Dorland B, et al. (2009) Proline affects brain function in 22q11DS children with the low activity COMT 158 allele. Neuropsychopharmacology 34: 739-746.

32. Kane JM, Leucht S, Carpenter D, Docherty JP (2003) The expert consensus guideline series. Optimizing pharmacologic treatment of psychotic disorders. Introduction: methods, commentary, and summary. J Clin Psychiatry 64 Suppl 12: $5-19$.

33. Kay SR, Fiszbein A, Opler LA (1987) The positive and negative syndrome scale (PANSS) for schizophrenia. Schizophr Bull 13: 261-276.

34. Canavan AGM, Dunn G, McMillan TM (1986) Principal components of the WAIS-R. Br J Clin Psychology 25: 81-86.

35. Wechsler D (1997) WAIS-III: Wechsler Adult Intelligence Scale. Administration and Scoring Manual. San Antonio: TX: Psychological Corporation.

36. Provencher SW (1993) Estimation of metabolite concentrations from localized in vivo proton NMR spectra. Magn Reson Med 30: 672-679.

37. Stone JM, Day F, Tsagaraki H, Valli I, McLean MA, et al. (2009) Glutamate dysfunction in people with prodromal symptoms of psychosis: relationship to gray matter volume. Biol Psychiatry 66: 533-539.

38. Kegeles LS, Shungu DC, Anjilvel S, Chan S, Ellis SP, et al. (2000) Hippocampal pathology in schizophrenia: magnetic resonance imaging and spectroscopy studies. Psychiatry Res 98: 163-175.

39. Lutkenhoff ES, van Erp TG, Thomas MA, Therman S, Manninen M, et al. (2008) Proton MRS in twin pairs discordant for schizophrenia. Mol Psychiatry. In press.

40. Van Elst LT, Valerius G, Buchert M, Thiel T, Rusch N, et al. (2005) Increased prefrontal and hippocampal glutamate concentration in schizophrenia: evidence from a magnetic resonance spectroscopy study. Biol Psychiatry 58: 724-730.

41. Olbrich HM, Valerius G, Rusch N, Buchert M, Thiel T, et al. (2008) Frontolimbic glutamate alterations in first episode schizophrenia: evidence from a magnetic resonance spectroscopy study. World J Biol Psychiatry 9: 59-63.

42. Delwing D, Sanna RJ, Wofchuk S, Wyse AT (2007) Proline promotes decrease in glutamate uptake in slices of cerebral cortex and hippocampus of rats. Life Sci 81: $1645-1650$.

43. Catani M, Cherubini A, Howard R, Tarducci R, Pelliccioli GP, et al. (2001) (1)H-MR spectroscopy differentiates mild cognitive impairment from normal brain aging. Neuroreport 12: 2315-2317.

44. Siger M, Schuff N, Zhu X, Miller BL, Weiner MW (2009) Regional myo-inositol concentration in mild cognitive impairment Using $1 \mathrm{H}$ magnetic resonance spectroscopic imaging. Alzheimer Dis Assoc Disord 23: 57-62.

45. Beacher F, Simmons A, Daly E, Prasher V, Adams C, et al. (2005) Hippocampal myo-inositol and cognitive ability in adults with Down syndrome: an in vivo proton magnetic resonance spectroscopy study. Arch Gen Psychiatry 62: 1360-1365.

46. Irvine RF, Schell MJ (2001) Back in the water: the return of the inositol phosphates. Nat Rev Mol Cell Biol 2: 327-338.
47. Fisher SK, Novak JE, Agranoff BW (2002) Inositol and higher inositol phosphates in neural tissues: homeostasis, metabolism and functional significance. J Neurochem 82: 736-754.

48. Schousboe A (2003) Role of astrocytes in the maintenance and modulation of glutamatergic and GABAergic neurotransmission. Neurochem Res 28: 347-352.

49. Danbolt NC (2001) Glutamate uptake. Prog Neurobiol 65: 1-105.

50. Chang L, Friedman J, Ernst T, Zhong K, Tsopelas ND, et al. (2007) Brain metabolite abnormalities in the white matter of elderly schizophrenic subjects: implication for glial dysfunction. Biol Psychiatry 62: 1396-1404.

51. Rusch N, Tebartz van EL, Valerius G, Buchert M, Thiel T, et al. (2008) Neurochemical and structural correlates of executive dysfunction in schizophrenia. Schizophr Res 99: 155-163.

52. Ohrmann P, Siegmund A, Suslow T, Pedersen A, Spitzberg K, et al. (2007) Cognitive impairment and in vivo metabolites in first-episode neuroleptic-naive and chronic medicated schizophrenic patients: a proton magnetic resonance spectroscopy study. J Psychiatr Res 41: 625-634.

53. Tayoshi S, Sumitani S, Taniguchi K, Shibuya-Tayoshi S, Numata S, et al. (2009) Metabolite changes and gender differences in schizophrenia using 3-Tesla proton magnetic resonance spectroscopy (1H-MRS). Schizophr Res 108: 69-77.

54. Kates WR, Miller AM, Abdulsabur N, Antshel KM, Conchelos J, et al. (2006) Temporal lobe anatomy and psychiatric symptoms in velocardiofacial syndrome (22q11.2 deletion syndrome). J Am Acad Child Adolesc Psychiatry 45: 587-595.

55. Eliez S, Blasey CM, Schmitt EJ, White CD, Hu D, et al. (2001) Velocardiofacial syndrome: are structural changes in the temporal and mesial temporal regions related to schizophrenia? Am J Psychiatry 158: 447-453.

56. Bloemen OJ, Gleich T, Koning MB, da Silva Alves F, de Haan L, et al. (2011) Hippocampal Glutamate Levels and Striatal Dopamine D(2/3) Receptor Occupancy in Subjects at Ultra High Risk of Psychosis. Biol Psychiatry. In press.

57. Stone JM, Howes OD, Egerton A, Kambeitz J, Allen P, et al. (2010) Altered relationship between hippocampal glutamate levels and striatal dopamine function in subjects at ultra high risk of psychosis. Biol Psychiatry 68: 599-602.

58. Perneger TV (1998) What's wrong with Bonferroni adjustments. BMJ 316: 1236-1238.

59. Coyle JT (2006) Glutamate and schizophrenia: beyond the dopamine hypothesis. Cell Mol Neurobiol 26: 365-384.

60. Lipska BK (2004) Using animal models to test a neurodevelopmental hypothesis of schizophrenia. J Psychiatry Neurosci 29: 282-286.

61. Goldman AL, Pezawas L, Mattay VS, Fischl B, Verchinski BA, et al. (2009) Widespread reductions of cortical thickness in schizophrenia and spectrum disorders and evidence of heritability. Arch Gen Psychiatry 66: 467-477.

62. Bertolino A, Callicott JH, Mattay VS, Weidenhammer KM, Rakow R, et al. (2001) The effect of treatment with antipsychotic drugs on brain Nacetylaspartate measures in patients with schizophrenia. Biol Psychiatry 49: 39-46. 